\title{
A topological multilayer model of the human body
}

\author{
Antonio Barbeito, ${ }^{1}$ Marco Painho, ${ }^{2}$ Pedro Cabral, ${ }^{2}$ João O'Neill ${ }^{3}$ \\ 'School of Technology and Management of Agueda, University of Aveiro, Agueda; ${ }^{2}$ Nova \\ Information Management School, Universidade Nova de Lisboa; ${ }^{3}$ Department of Anatomy, \\ Universidade Nova de Lisboa, Lisbon, Portugal
}

\begin{abstract}
Geographical information systems deal with spatial databases in which topological models are described with alphanumeric information. Its graphical interfaces implement the multilayer concept and provide powerful interaction tools. In this study, we apply these concepts to the human body creating a representation that would allow an interactive, precise, and detailed anatomical study. A vector surface component of the human body is built using a three-dimensional (3D) reconstruction methodology. This multilayer concept is implemented by associating raster components with the corresponding vector surfaces, which include neighbourhood topology enabling spatial analysis. A root mean square error of $0.18 \mathrm{~mm}$ validated the threedimensional reconstruction technique of internal anatomical structures. The expansion of the identification and the development of a neighbourhood analysis function are the new tools provided in this model.
\end{abstract}

\section{Introduction}

Image segmentation is used in different areas and with different

Correspondence: Antonio Barbeito, School of Technology and Management of Agueda, University of Aveiro, Apartado 473, 3754-909, Agueda, Portugal. Tel. +35.123.4611500.

E-mail: amtb@ua.pt

Key words: 3D GIS; Segmentation; Topology; Spatial analysis.

Acknowledgements: the authors acknowledge the contribution of the U.S. National Library of Medicine for making available the datasets used in this work.

Received for publication: 13 May 2015.

Revision received: 27 August 2015.

Accepted for publication: 30 August 2015.

(C) Copyright A. Barbeito et al., 2015

Licensee PAGEPress, Italy

Geospatial Health 2015; 10:375

doi:10.4081/gh.2015.375

This article is distributed under the terms of the Creative Commons Attribution Noncommercial License (by-nc 3.0) which permits any noncommercial use, distribution, and reproduction in any medium, provided the original author(s) and source are credited. types of images. The segmentation of human body structures with red, green and blue (RGB) images has been the subject of research in several works (Schiemann et al., 1997; Pommert et al., 2001; Flores and Schmitt 2005; Liu et al., 2014) associated with the Visible Human Project (VHP) (http://www.nlm.nih.gov/research/visible/ animations.html) (Spitzer et al., 1996; Park et al., 2006; Zhang et al., 2006). Other methods, such as RGB cryosections (i.e. images slices), computed tomography (CT) and magnetic resonance imaging (MRI) use different combinations of image types included in these projects (Imielińska et al., 2000a, 2000b; Xue et al., 2014). The joint use of different types of image for segmentation was chosen to take advantage of each format characteristics and to overcome identified, specific limitations. RGB images make available quite realistic information about the visual aspect of the anatomical structures. The quantity of stored information, larger than CT and MRI (Takanashi et al., 2002), enable a greater level of discrimination among these structures. However, RGB images present drawbacks when it comes to the delineate bones with articulations and/or tendons. In these cases, CT images are more efficient (Beylot et al., 1996).

Regardless of the input data used, the segmentation procedures can be manual, semiautomatic or automatic. The automatic procedure cannot be considered when the segmentation needs a high level of detail and non-ambiguity is important (Schiemann et al., 1997; Beylot et al., 1996; Riemer et al., 2007). This occurs when a high level of quality in the segmentation delineation is required to reconstruct the anatomical structures. The semiautomatic methods use the automatic components to facilitate and accelerate the segmentation procedure and the manual intervention to define parameters, correct errors and perform quality control (Schiemann et al., 1997; Takanashi et al., 2002; Imelińska et al., 2000a, 2000b; Beveridge et al., 2013).

Geographical information systems (GIS) operate with spatial data models associated with alphanumeric information. Traditionally, these systems are more focused on the representation of objects more related to the Earth than anatomical features. However, the inclusion of human-body models in a GIS environment would greatly benefit from the characteristics of this approach to relate three-dimensional (3-D) structures to each other. Indeed, a GIS-based map of the vertebral canal was recently produced to spatially guide veterinary surgeons when evaluating extruded disc herniation in dogs, a common problem in this animal (Daraban et al., 2014).

This work addresses two GIS characteristics: the multilayer concept and topology. The multilayer concept consists in structuring the information into layers and is implemented in the table of contents (TOC) of the interface. From the TOC, one can act independently over information layers to make them accessible to the operations to be carried out. Topology, on the other hand, concerns the study of the relationships among the spatial objects that compose the model. These relationships can be obtained in two ways (Ellul and Haklay 2006): i) impromptu, the relationships among objects is determined when need- 
ed; and ii) pre-calculated, the topological relationships are explicitly described in a topological data model.

Thus, analytic functions that use topology do not require the use of a topological model. The importance of topological models in GIS is associated with data validation operations and spatial analysis procedures (Raper and Maguire, 1992). In the absence of topological models, the implementation of analysis that requires topology implies the establishment of ad hoc topological relationships, making them more complex and time-consuming. In two-dimensional (2-D) GIS, the topological relationships are well exploited and lead to the development of spatial analysis tools. The conversion to 3-D GIS entails increased complexity of data models and analytical functions (Abdul-Rahman and Pilouk, 2008). The greater complexity of 3-D models is also present on topological relationships since in a 3-D space, new and more complex relationships among objects emerge. The study of topological relations is often based on the 9-intersection model (9-IM) (Egenhofer and Herring, 1990) which identifies the relationship between two objects through the intersections between the interior, exterior and border. The implementation of 9-IM in spatial databases reflects the greater complexity of topological relationships in 3-D. While the 2-D topological relations have been constantly improved in spatial databases, the development of their 3-D equivalents has been slow (Xu and Zlatanova, 2013). However, with the progress in 3-D GIS, the emphasis is shifting from visualisation to querying and analysis (Penninga and van Oosterom, 2008). From a GIS perspective, explicit topological models are required when the maintenance and spatial analysis become key aspects in 3-D GIS.

The representation of the human body can use vector, raster or integrated (i.e. combined vector and raster) models. While the vector models are used to represent the surfaces of anatomical structures (Visible Body, 2008), the raster models allow the representation of the interior of those structures (Höhne, 2009). The use of integrated models enables the representation of the surface and of the inside of anatomical structures. They also facilitate the development of topological information (Barbeito et al., 2014). As in GIS models, the purpose of the representations of the human body cannot be only dedicated to visualisation. It is also important to make functions available, e.g. identification of anatomical structures, querying and analytical operations. Since spatial analysis is a fundamental aspect to consider, topology, as implemented in the GIS, is also of great importance.

This work has as main objective the development of an innovative system consisting of an integrated topological model of the human body, a graphical interface and tools that allow the interaction and study of the model. The system implements GIS capabilities for structuring information and handling topological models, which is accomplished by developing an application that reproduces the capabilities of a 3-D anatomical atlas. It is, therefore, an application with didactic purposes for use in the study of human anatomy.

\section{Materials and Methods}

\section{Data}

This work uses RGB and CT cryosection images (slices) from the VHP (Figure 1) as input data and an integrated human-body model (Barbeito et al. 2014). RGB images are a sequence of axial planes of a male human body with 24-bit of depth colour, spaced $1 \mathrm{~mm}$ along the $\mathrm{Z}$ axis, and a pixel of $0.33 \mathrm{~mm}$ in the XY plane. The CT data consists of axial CT scans taken at $1 \mathrm{~mm}$ intervals with a resolution of 512 pixels by 512 pixels (a pixel of $1 \mathrm{~mm}$ ). These are 12 bits greyscale images.
The human-body integrated model is constituted by a raster-based volume and a vector-based surface. The former is composed by two different structures: a 3-D RGB matrix constituted by voxels, i.e. 3 containing true colour information of anatomical structures and a layer matrix (LM) which discriminates the anatomical structures. The LM was generated by segmentation of the anatomical structures. The surface vector component is constituted by a mesh of triangles which is the outer surface of the body. This was built from the point cloud formed by the coordinates of the LM boundary voxels (the smallest distinguishable box-shaped parts of a 3-D image, i.e. the 3-D counterpart to a pixel) using the Screened Poisson algorithm (Kazhdan and Hoppe, 2013).

Although the anatomical structures are segmented, the multilayer concept was not fully implemented in the input model since there are no corresponding surface vector components for the several raster-segmented components. Figure 2 shows the flow chart of the procedure for obtaining this model and its various components.

\section{Adjustment and segmentation of computed tomogra- phy and red green blue images}

The segmentation of the input model depends on a semiautomatic procedure that works on RGB images. In this method, the automatic component allows accelerating the segmentation while the manual component is targeted at correcting the segmentation errors and controlling overall quality. In this semi-automatic implementation, one aspect which required manual intervention, and hence a greater expenditure of time, was the segmentation of the hard tissues in the neighbourhood of other structures with similar RGB values, such as tendons. In these cases, the segmentation algorithm presents difficulties in discriminating between the two structures.

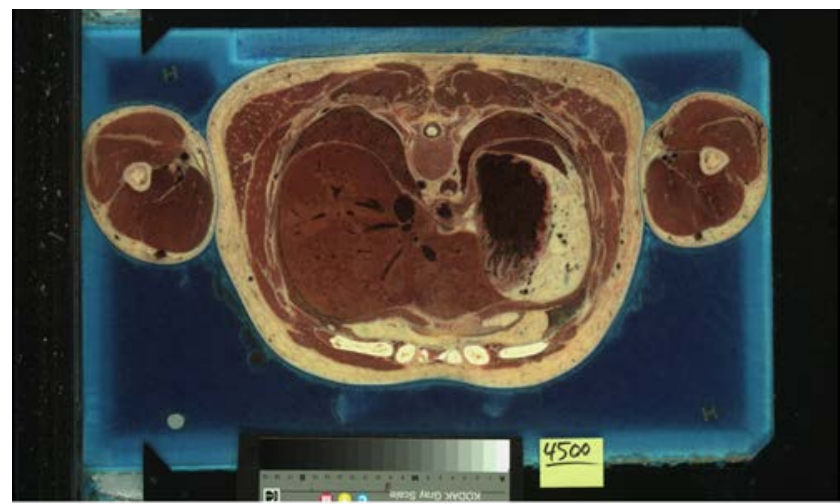

(a)

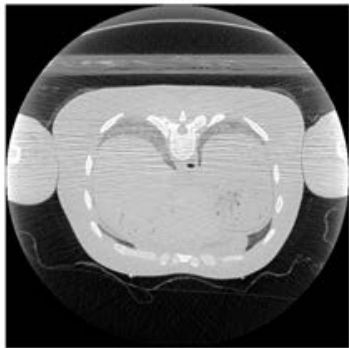

(b)

Figure 1. Data from the Visible Human Project: a) anatomical red, green and blue cryosection image; b) computed tomography image. 
To improve the functioning and minimising of the segmentation time, routines that operate and integrate the CT images on the application were developed. The segmentation of structures with CT images starts with the interactive setting of a threshold value over the target image. After initial segmentation, the programme evolves through the image sequence maintaining the threshold value that can be interactively adjusted.

In the case noise is present in the system, filters are provided to correct the segmentation areas. The procedure comprises applying the threshold value, filling gaps within the target area, eliminating islands (noise which consists of small spots occurring in the image) whose size can be adjusted and boundaries smoothed. The smoothing of the segmentation boundaries aims at minimising the pixelisation effect that occurs in the reconstruction of the final 3-D model.

Since the RGB and CT images have different sizes and resolution, their adjustment is made through an interactive definition of the translation parameters and a scale factor. These parameters are determined to convert the CT images into the RGB image coordinate system. Through an interface where the images are overlaid, the visual validation of the CT image segmentation on the RGB image is carried out interactively.

\section{3-dimensional reconstruction of the surface vector component}

The reconstruction of the surface vector components follows the methodology previously used (Barbeito et al., 2014) in which the voxel coordinates of the boundary extracted from the LM matrix are used to generate a point cloud. From this cloud, through the application of a three-dimensional reconstruction Screened Poisson algorithm and added filtering to smooth the surface, the mesh of triangles that constitutes the reconstructed surface structure, is generated. This structure receives an identifier (ID) identical to the one assigned to the respective raster internal component that is described in LM.

Two files with colour information were included in the model structure. This information may be used to visualise the anatomical structures in the graphical user interface. The first file associates a single colour with each segmented structure, while the second associates each of the defined colours with a group of structures, such as muscles or bones.

The 3-D reconstruction involves the application of several tools which, from the initial masks, produce a final vector model. To assess the compliance of the vector reconstruction with the input data, a procedure was developed to validate the methodology and apply it to the various segmented anatomical structures. The validation process was performed on the vector model corresponding to the outer surface of the body in question. This process determines a parameter that measures the gap between the peripheral region of the body in axial images and the corresponding vector model obtained by the 3 -D reconstruction. The parameter used was the root mean squared error (RMSE) that provides a measure of the difference between the two entities and by comparing the value obtained with reference values. Thus, it is possible to assess the positional quality of the vector model with regard to the input data.

The determination of RMSE value is based on the calculation of distances, $\mathrm{d}_{\mathrm{j}}$, between the points of the vector component $\left(x_{i}^{v}, y_{\mathrm{i}}^{v}\right)$, and the corresponding points on the LM border $\left(x_{i}^{r}, y_{i}^{r}\right)$. The centroids of the triangles were considered for the points of the vector component. The dots at the LM border were calculated by linear interpolation to obtain the voxel coordinates that should be at the same level of the considered $\mathrm{Z}$ centroid.

Since the original data are images composed of pixels, the deviations of the vector model were evaluated in terms of the size of such data. As we are dealing with a 3-D model in this case, the comparison was made in terms of the voxel size. However, as the resulting voxels of the input data are not cubic, three different dimensions were considered to generate values for RMSE comparison: the voxel length in the $\mathrm{XY}$ plane, the voxel length in the direction $\mathrm{Z}$ and the side of a conceptual cubic voxel with the same volume as the original voxel. Given the values of the dimensions considered, the pixel side in the $\mathrm{XY}$ plane, being the smallest of the three, leads to a more unfavourable comparison based on the RMSE. The reference value to validate the vector model was 1.00 in terms of voxel size (Pouncey et al., 1999). The final comparison was made between this reference value and the value obtained for the RMSE divided by each of the dimensions considered.

\section{Topological model}

The input model comprises containment of the topological information resulting from the partition of space by the LM voxels. The expansion of the topological structure of the model consisted in establishing neighbourhood relationships at the level of the surface vector compo-

Table 1. Root mean square error value assessment measured between the vector surface and the boundary of the respective raster component.

$\begin{array}{ll}\text { Reference } & \text { RMSE/reference ratio }\end{array}$

Side of the original voxel along the $\mathrm{Z}$ axis

0.180

Side of the cubic voxel $\quad 0.375$

\begin{tabular}{ll}
\hline Side of original the voxel on the XY plane & 0.545
\end{tabular}

RMSE, root mean square error.

Table 2. Determination of the left leg muscles that are neighbours of a predetermined structure.

\begin{tabular}{|c|c|c|}
\hline Structure & Analysis result (muscles) & Running time (sec) \\
\hline Femur & $\begin{array}{c}\text { Adductor brevis, Adductor magnus, Gluteus medius, Obturator externus, } \\
\text { Quadratus femoris, Biceps femoris, Vastus intermedius, } \\
\text { Vastus lateralis, Vastus medialis }\end{array}$ & $0.04 / 8.05$ \\
\hline Rectus femoris & Sartorius, Tensor fasciae latae, Vastus intermedius, Vastus lateralis, Vastus medialis & $0.04 / 8.38$ \\
\hline Vastus lateralis & Rectus femoris, Tensor fasciae latae, Vastus intermedius & $0.04 / 14.11$ \\
\hline
\end{tabular}


nents. To this end, all the surrounding structures were identified for each frame. The decision on whether two structures are neighbours takes into account the size of the voxels of the input data, i.e. for each structure, the points that form the vertices of the triangles of the vector component are considered, and for each vertex, the points that are at a distance comparable to the voxel size and do not belong to the same structure, are determined. These points are considered neighbours and the neighbouring structures are registered for each of them. After checking all points of a given structure, a list is saved without repetition of the surrounding structures. The procedure is performed for all the structures and the information is explicitly described in the model by associating each ID of the structure with the ID of the neighbouring structures. The sequence of operations that lead to the production of the expanded model is represented in Figure 2.

\section{New functionalities}

From the development of a multilayer system and a neighbourhood topology, two new features were developed. The first is an identification function that extends the capability of the input model identification tool. In the input model, this feature was applied only to the raster component. In the expanded model, it was possible to display one or more structures individually and to identify them interactively on the vector surface. The second feature is a spatial neighbourhood analysis function, which determines the neighbouring structures of a pre-determined structure. The first step to implement this function is to identify the pre-determined anatomical structure. It is also possible to further select a subset of structures, inside which the surrounding structures must be evaluated. The result of this analysis was obtained by the intersection of this set with the set of the neighbouring structures that, in turn, can be read in the table that contains information about the neighbourhood.

\section{Results}

\section{Computed tomography image segmentation}

Computed tomography image segmentation is based on applying a threshold value to highlight the hard tissues, a principle complemented with other procedures to further improve the final result. The segmentation module has been enriched with additional functions to eliminate noise. The result of the segmentation of each anatomical structure is a set of binary images with the value of 1 in the structure and zero outside. After the segmentation of a CT image, the result is transformed into a coordinate system common to the RGB images. The option to transform the coordinate system of CT images to RGB images has to do with the fact that the latter is already integrated with the application. The transformation parameters are determined interactively by translations in the $\mathrm{X}$ and $\mathrm{Y}$ directions and a scale factor.

\section{Reconstruction of the surface vector components of anatomical structures}

The calculation of the RMSE used to validate the reconstruction method involved 14,389 centroids of the triangles that compose the vector component of the entire body surface. The obtained value was 0.18 $\mathrm{mm}$ (Table 1). Once reconstructed, the surface vector components of the segmented anatomical structures are stored in the model structure together with the corresponding IDs entered in the LM matrix as well as the assigned colour information (Figure 3 ).

\section{Topology and functions}

The implementation of the multilayer system from the vector components of each segmented structure is made by building a TOC in the graphical interface, which lists all segmented structures and allows, through check boxes, to enable or disable layers for visualisation.

Using the multilayer system and the identification function, it is possible to act on the vector component, identifying each structure represented in the graphical user interface. Note that this feature maintains the ability to identify structures on the raster components disclosed by section planes as in the input model.

In the developed system, the neighbourhood analysis is based on the same type of topology explicitly described in the model. Such analyses were carried out, with and without the topological information, on

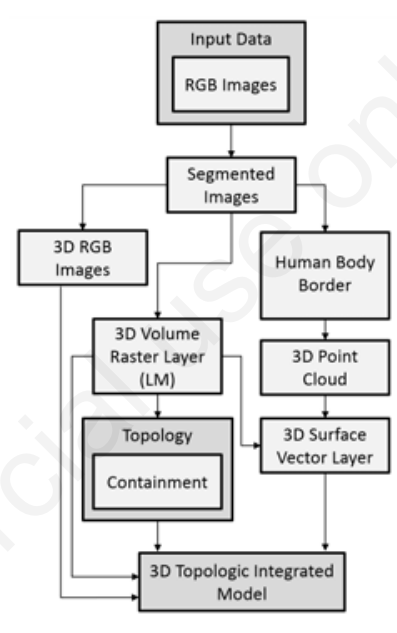

(a)

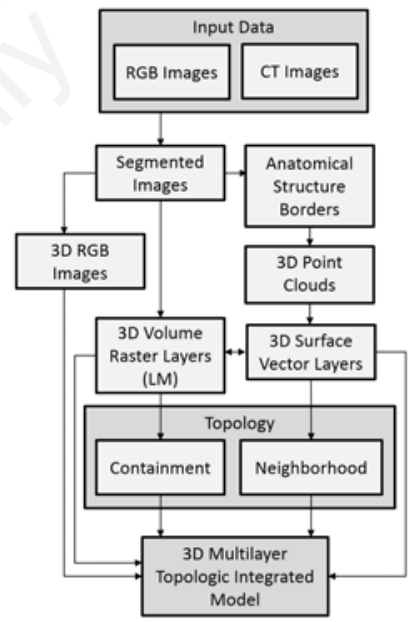

(b)
Figure 2. Methodological workflow for developing the integrated models: a) input model; b) expanded model with the inclusion of neighbourhood topology.

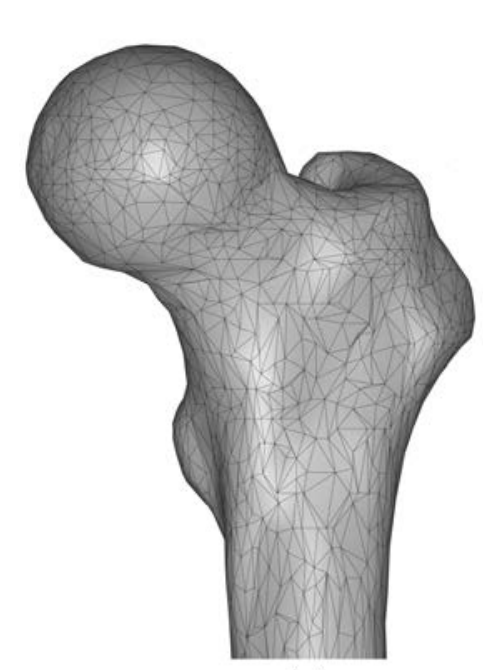

(a)

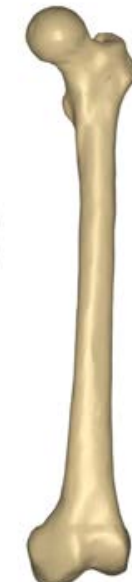

(b)

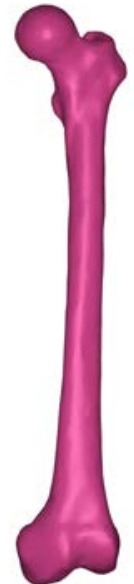

(c)
Figure 3. Reconstruction of the vector surface component of the left femur: a) triangle mesh that materialises the geometrical component; b) false colour assigned to the bones; c) colour assigned to the segmentation of the left femur. 
three anatomical structures (Femur, Musculus rectus femoris and Vastus lateralis). The results and the run times of analyses are shown in Table 2. Figure 4 depicts the final application, including tools and the graphical interface.

\section{Discussion}

This paper presents a system to represent and explore the human body. It consists of an integrated multilayer topological model, a graphical user interface and new tools. The system implementation includes the improvement of a semiautomatic segmentation application that now includes the ability to segment CT images, the expansion of surface vector and topological components of the model and the development of identification and spatial neighbourhood analysis functions.

The 3-D surface is constructed by applying the Screened Poisson reconstruction algorithm. It is composed by vector triangle meshes representing the outer surfaces of the anatomical structures. The contribution of these vector components to the input data was evaluated by the RMSE parameter. The ratio between the RMSE value and the raster sizes is significantly less than 1.00 , the benchmark used to perform this validation even in the most unfavourable situation, i.e. the comparison of the RMSE value with the pixel size in the XY plane, which means that the vector component surface receives a suitable geometric adjustment with respect to the original raster data. This result is achieved on a model that was obtained after applying the Poisson Screen algorithm and filtering methods. The intention of adding the latter component was to smooth the final surface allowing mitigation of the pixelisation effect. The use of filtering also enabled the visual model validation.

In the input model, the 3-D representation based on the surface vector component was only possible at the level of the entire human body, which made it possible to introduce cutting plans allowing inspection of the inside of the body. The current model allows obtaining 3-D representations and defines cutting planes at each anatomical structure level. This feature deepens the operating capacity of the exploration of the human body. In the input model, when defining a cutting plane, it was not possible to perceive the three-dimensional morphology of the internal structures. This lack of perception can be minimised by performing cutting planes for this structure using different perspectives. However, the existence of the surface vector component and the multilayer structure allows, beyond the cross-sectional information, the three-dimensional reconstruction and visualisation of anatomical structures, i.e. it is possible to directly observe its true shape. Figure 5 shows the application of a cutting plane in the initial model and some of the increased possibilities for the expanded model.

One of the advantages of the topologic models is the gain of time in spatial analysis operations. As can be seen in Table 2, the non-inclusion of topology in the model led to a run-time, which was254.5 times higher on average. Note that the results in Table 2 refer only to the segmented structures in the left leg (bones and muscles).

Despite having been developed as a system for didactic purposes, since their query and analysis functions depend basically on the structure of the final model, this application can be used with other models, e.g., from CT imagery. To this end, the reconstruction tools of the application itself may be used. Thus, it is possible to expand the scope of the current application to the clinical context.

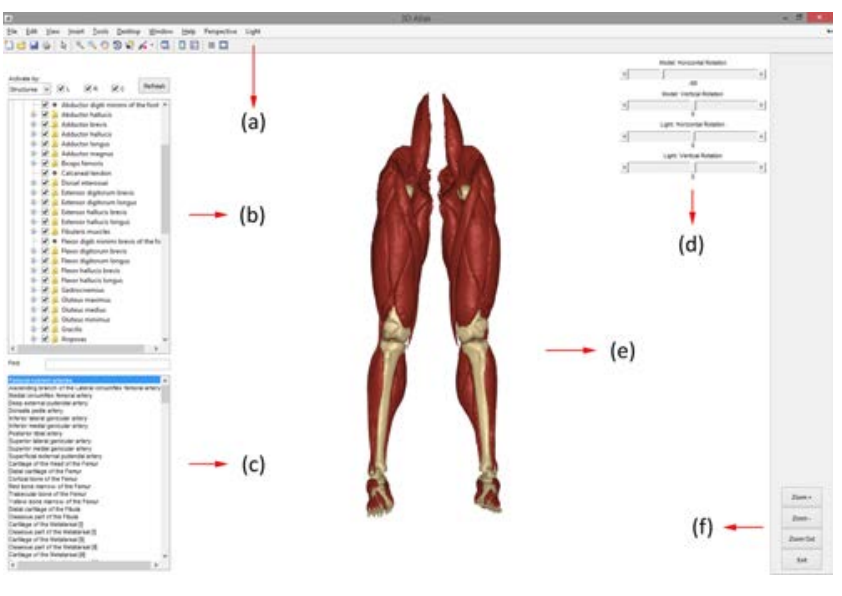

Figure 4. Application functional areas : a) menu including predefined perspectives and light positions; $b$ ) table of contents region where the anatomical structures are selected; c) structures represented; d) tools to rotate the model and the incident light; e) graphical area (representing the lower limb); f) zooming and exit buttons.

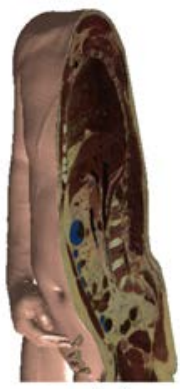

(a)

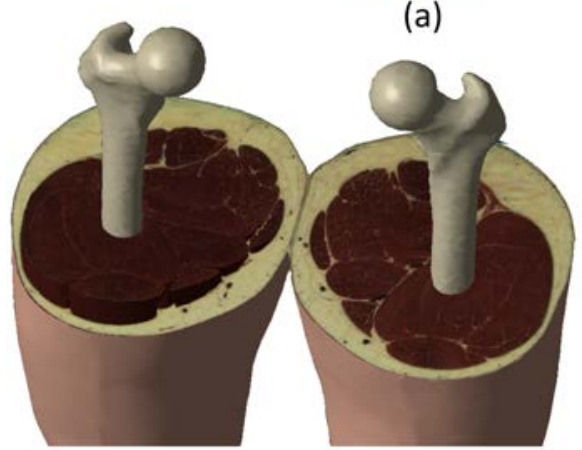

(b)

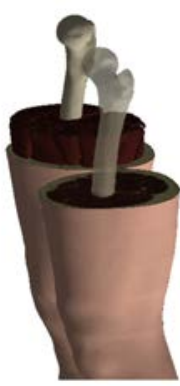

(c)
Figure 5. Views in the input and expanded models: a) in the input model, the cutting planes are made on the body's outer surface; b) in the expanded model, it is possible to represent threedimensionally each of the segmented structures, on which it is possible to set different cutting plans; c) the multilayer structure allows the assignment of different properties to be preselected for various structures, e.g. setting the degree of transparency. 


\section{Conclusions}

The current model comprises containment and neighbourhood topological components, which were used to develop spatial analysis functions. However, a connectivity topological component is still missing in the model. This could be used to develop measurement tools over linear structures that have branches, such as the length measurement of a blood vase. Other features to be developed include texturing surface vector components with true colour to allow more realistic visualisations, volume and surface measurement tools of the anatomical structures, and identification functions with increasing levels of detail.

\section{References}

Abdul-Rahman A, Pilouk M, 2008. Spatial data modelling for 3D GIS. Springer, Berlin, Germany.

Barbeito A, Cabral P, Painho M, O’Neill J, 2014. An integrated model of the human body. In: Proc. 14th Conference on Health Information Systems, Santarém, Portugal.

Beveridge E, Minhua Ma, Rea P, Bale K, Anderson P, 2013. 3D visualisation for education, diagnosis and treatment of lliotibial band syndrome. In: Proc.of 2013 Int. Conf. of the Computer Medical Applications (ICCMA), Sousse, Tunisia, pp 1-6.

Beylot P, Gingins P, Kalra P, Magnenat Thalmann N, Maurel W, Thalmann D, Fasel J, 1996. 3D interactive topological modeling using visible human dataset. Comput Graph Forum 15:33-44.

Daraban C, Murino C, Marzatico G, Mennonna G, Fatone G, Auletta L, Miceli F, Vulpe V, Meomartino L, 2014. Using geographical information system for spatial evaluation of canine extruded disc herniation. Geospat Health 9:213-20.

Egenhofer MJ, Herring J, 1990. A mathematical framework for the definition of topological relationships. In Proc. of the 4th Int. Symp. on Spatial Data Handling, Zurich, Switzerland, pp 803-13.

Ellul C, Haklay M, 2006. Requirements for topology in 3D GIS. Trans GIS 10:157-75.

Flores JM, Schmitt F, 2005. Segmentation, reconstruction and visualization of the pulmonary artery and the pulmonary vein from anatomical images of the visible human project. In: Proc. of the 6th Mexican Int. Conf. on Computer Science, IEEE Computer Society, Washington, DC, USA, pp 136-44.

Höhne KH, 2009. Voxel-Man 3D-Navigator: brain and skull; regional, functional, and radiological anatomy. Springer, Berlin, Germany.

Imelińska C, Downes MS, Yuan W, 2000a. Semi-automated color segmentation of anatomical tissue. Comput Med Imag Grap 24:173-80.
Imelińska C, Metaxas D, Udupa J, Jin Y, Chen T, 2000b. Hybrid segmentation of the visible human data. Available from: $\mathrm{ftp} / / / \mathrm{ftp}$.cis.upenn.edu/pub/dnm/micai-update.doc

Kazhdan M, Hoppe H, 2013. Screened poisson surface reconstruction. ACM T Graphic 32:1-13.

Liu B, Li H, Jia X, Zhao X, Liu Z, Zhao Q, Zhang H, 2014. A simple method of rapid and automatic color image segmentation for serialized visible human slices. Comput Electr Eng 40:870-83.

Park, JS, Chung MS, Hwang SB, Byeong Seok Shin BS, Park HS, 2006. Visible Korean human: its techniques and applications. Clin Anat 19:216-24.

Penninga F, van 0osterom P, 2008. First implementation results and open issues on the Poincare-TEN data structure. In: Peter van Oosterom P, Zlatanova S, Penninga F, Fendel EM, eds. Advances in 3D geoinformation systems. Springer, Berlin-Heidelberg, Germany, pp 177-97.

Pommert A, Höhne KH, Pflesser B, Richter E, Riemer M, Schiemann T, Schubert R, Schumacher U, Tiede U, 2001. Creating a high-resolution spatial/symbolic model of the inner organs based on the visible human. Med Image Anal 5:221-8.

Pouncey R, Swanson K, Hart K, 1999. ERDAS field guide ${ }^{\mathrm{TM}}$. Erdas, Inc., Atlanta, GA, USA.

Raper JF, Maguire DJ, 1992. Design models and functionality in GIS. Comput Geosci 18:387-94.

Riemer M, Park JS, Chung MS, Handels H, 2007. Improving the 3D visualization of the visible korean human via data driven 3D segmentation in RGB color space. In: Magjarevic R and Nagel JH, eds. World congress on medical physics and biomedical engineering 2006. Springer Berlin, Gemany, pp 4200-3.

Schiemann T, Tiede U, Höhne KH, 1997. Segmentation of the visible human for high-quality volume-based visualization. Med Image Anal 1:263-70.

Spitzer V, Ackerman MJ, Scherzinger AL, Whitlock D, 1996. The visible human male: a technical report. J Am Med Inform Assn 3:118-30.

Takanashi I, Lum E, Ma K-L, Meyer J, Hamann B, Olson AJ, 2002. Segmentation and 3D visualization of high-resolution human brain cryosections. Available from: jmeyer.dyndnshome.com/PAPERS/c-14.pdf

Visible $\quad$ Body, 2008. Available from: http://www.visiblebody.com/index.html

Xu D, Zlatanova S, 2013. An approach to develop 3d Geo-DBMS topological operators by re-using existing $2 \mathrm{~d}$ operators. In: Proc. of the 8th 3DGeoInfo Conf. \& WG II/2 Workshop, Istanbul, Turkey, pp 291-8.

Xue Z, Antani S, Long LR, Demner-Fushman D, Thoma GR, 2014. Body segment classification for visible human cross section slices. In: Proc. of the 27th International Symposium on Computer-Based Medical Systems (CBMS), New York, NY, USA, pp 199-204.

Zhang S-X, Heng P-A, Liu Z-J, 2006. Chinese visible human project. Clin Anat 19:204-15. 